\title{
Expression of FOXA1 Is Associated with the Tumor-Infiltrating M2 Macrophage, Cytotoxic T Lymphocyte, and Effect of Chemotherapy in Bladder Cancer
}

\author{
Yiduo Wang ${ }^{a, b}$ Huan $\mathrm{Qu}^{c}$ Bin $\mathrm{Xu}^{\mathrm{a}, \mathrm{b}}$ Jianping Wu${ }^{\mathrm{a}, \mathrm{b}}$ Kai Lu ${ }^{\mathrm{a}, \mathrm{b}}$ Chunhui Liu ${ }^{\mathrm{a}, \mathrm{b}}$ \\ Shuqiu Chen ${ }^{a, b}$ Ming Chen ${ }^{a, b}$ \\ ${ }^{a}$ Department of Urology, Zhongda Hospital, School of Medicine, Southeast University, Nanjing, China; ${ }^{b}$ Institute of \\ Urology, Surgical Research Center, Southeast University Medical School, Nanjing, China; ${ }^{C H}$ ealth Management Center, \\ Zhongda Hospital, Southeast University, Nanjing, China
}

\section{Keywords}

Bladder cancer · FOXA1 - M2 tumor-associated macrophage · Cytotoxic T lymphocyte - Chemotherapy

\begin{abstract}
Purpose: Cisplatin-containing combination chemotherapy has been the standard of care since the late 1980s, but the response rate is $<50 \%$. Studies have shown that the efficiency of chemotherapy differs among molecular subtypes of bladder cancer. In this study, we aimed to correlate FOXA1, a marker for differentiation of the basal and luminal subtypes, with tumor immune cell infiltration and the effect of chemotherapy in bladder cancer. Materials and Methods: Eighty-three patients with bladder cancer treated with chemotherapy were reviewed. Clinicopathological variables for each case were recorded. FOXA1, M2 tumor-associated macrophage (TAM), dendritic cell (DC), and cytotoxic T lymphocyte $(C T L)$ were examined by immunohistochemistry. The relationship between FOXA1, immune cell infiltration, and clinical response to chemotherapy was assessed. Results: The overall objective response rate was $34 \%$. The objective response rate for tumors with lower FOXA1 expression was $58 \%$ and for tumors with higher FOXA1 expression was $12 \%$. Tumors with infiltrated M2 TAM proportion $<3 \%$ had a high-
\end{abstract}

karger@karger.com www.karger.com/uin

Karger $\stackrel{\text { ' }}{5}$

GOPEN ACCESS
(C) 2021 The Author(s)

Published by S. Karger AG, Basel

This is an Open Access article licensed under the Creative Commons Attribution-NonCommercial-4.0 International License (CC BY-NC) (http://www.karger.com/Services/OpenAccessLicense), applicable to the online version of the article only. Usage and distribution for commercial purposes requires written permission. er objective response rate compared with infiltrated $\mathrm{M} 2 \mathrm{TAM}$ proportion $>3 \%$ tumors ( $46 \%$ vs. $21 \%, p=0.02$ ). Tumors with infiltrated CTL proportion $>5 \%$ had a higher objective response rate compared with infiltrated CTL proportion $<5 \%$ tumors ( $50 \%$ vs. $17 \%, p=0.002$ ). DCs showed no significant differences. We found that the objective response rate for tumors with lower FOXA1 expression, proportion $<3 \% \mathrm{M} 2$ TAM infiltration, and proportion $>5 \%$ CTL infiltration is $82 \%$. Lower FOXA1 expression was associated with low M2 TAM infiltration and high CTL infiltration. Conclusions: Thus, we showed that in patients with bladder cancer who received chemotherapy, the higher clinical response rate is associated with low FOXA1 expression, low M2 TAM infiltration, and high CTL infiltration.
(C) 2021 The Author(s)

Published by S. Karger AG, Basel

\section{Introduction}

Bladder cancer (BC) is the 7th most commonly diagnosed cancer in males and 11th in both genders [1]. It is estimated to cause 150,000 deaths per year [2]. Approximately $25 \%$ of $\mathrm{BC}$ patients are diagnosed with muscleinvasive bladder cancer (MIBC), with worse prognosis [3]. In the past 30 years, MIBC has been treated primar-

Correspondence to:

Ming Chen, mingchenseu@163.com 
ily by cisplatin-based combination chemotherapy and surgery [4].

Now, cisplatin-based chemotherapy is still the first-line treatment for patients with advanced BC who are not eligible for surgery [5-7]. But, its administration according to standard indications does not always lead to tumor regression and is associated with significant toxicity $[8,9]$. Patients who have progressive disease after the first-line chemotherapy have a poor prognosis [10]. The Cancer Genome Atlas (TCGA) defines the molecular subtypes of MIBC into at least 2 broad subtypes, luminal and basal, based on gene expression profiles [11]. Compared with the luminal subtype, basal-subtype MIBC is more sensitive to chemotherapy and shows a "hot" immunophenotype [11]. The expression of FOXA1 in MIBC is one of the important markers for the differentiation of molecular subtypes.

$\mathrm{BC}$ is known to have a high level of infiltrating immune cells and substantial adaptive immune response. Studies on tumor immune environment found that the presence of tumor-infiltrating immune cells may predict the prognosis and the effect on chemotherapy or immunotherapy. Recent works have shown that the presence of tumor-infiltrating lymphocytes, specifically cytotoxic $\mathrm{T}$ lymphocyte (CTL), correlates with a favorable prognosis and better response to chemotherapy $[12,13]$. Our objective was to conduct an evaluation to correlate the expression of FOXA1 and tumor-infiltrating lymphocytes with the response to chemotherapy.

\section{Materials and Methods}

\section{Patient Materials}

Between 2015 and 2018 at the Department of Urology, Zhongda Hospital, Southeast University, this retrospective study included patients with MIBC (pT2-4) who underwent neoadjuvant chemotherapy prior to radical cystectomy and standard pelvic lymph node dissection or who received chemotherapy alone $(n=83)$. This study was approved by the Ethics Committee of Zhongda Hospital, Southeast University (Approval No. 2018ZDKYSB102). All subjects provided written informed consent following the Declaration of Helsinki. Patients received 2-6 courses of cisplatin-based chemotherapy in the following schemes: gemcitabine (at a dose of $1,000 \mathrm{mg}$ per square meter) and cisplatin (at a dose of $70 \mathrm{mg}$ per square meter), all administered intravenously every 3 weeks. The clinical effect was evaluated with a CT scan after each 2 courses. For patients who were eligible for radical cystectomy, if tumor reduction after 2 courses was $>30 \%$, patients received 2 more courses to increase the effect. If tumor reduction was $<30 \%$ after 2 courses of chemotherapy, the patients were prescribed a radical cystectomy.

\section{Evaluation of Chemotherapy}

The clinical efficacy of chemotherapy was assessed by criteria of the WHO and the International Union Against Cancer based on the results of the CT scan. Complete response (100\% of tumor reduction), partial response (decrease in tumor diameter by $>50 \%$ ), stable disease (decrease in diameter by $<50 \%$ or no $>25 \%$ of increase), and progression disease (increase in tumor diameter by $>25 \%$ ) were registered. Patients with complete and partial response comprised the group with objective response, and patients with stabilization or progression comprised the group with the absence of response to chemotherapy.

\section{Immunohistochemistry}

A total of 83 MIBC specimens were obtained from Zhongda Hospital. All specimens were from patients undergoing radical cystectomy or transurethral resection of bladder tumor. Original sections of cystectomy specimens were collected and re-evaluated by an experienced uropathologist. Primary tumors were graded according to the WHO 1999 and TNM 2010 classifications. A series of 5- $\mu \mathrm{m}$ sections prepared from formalin-fixed paraffin-embedded tumor tissue blocks were stained according to the DAKO Envision system. Immunostaining results were evaluated independently by 2 authors. FOXA1 was localized using the FOXA1 antibody (RRID: ab5089; Abcam). The FOXA1 expression level was defined as follows: low expression $=$ no or faint staining of tumor cells and high expression level = intense staining of tumor cells (Fig. 1a). CD8 (RRID: ab17147; Abcam), HLA-DR (RRID: ab92511; Abcam), and CD163 (RRID: ab182422; Abcam) antibodies were used to detect CTL, dendritic cell (DC), and M2 tumorassociated macrophage (TAM), respectively. For systematic counting of immune cells, 6 ocular fields within a tissue were randomly chosen under a microscope at $\times 400$ magnification. The mean amount of positively stained immune cells was recorded.

\section{Statistics}

Data analysis was carried out using unpaired $t$ test, Fisher's exact test, and $\chi^{2}$ test. Significance was defined as $p<0.05$.

\section{Results}

A total of 83 patients ( 68 male and 15 female) were included in the present study, and their characteristics are shown in Table 1. Of the 83 patients, 24 (29\%) patients received neoadjuvant chemotherapy and 59 (71\%) received chemotherapy alone. After treatment, the overall objective response rate (complete response + partial response) was $34 \%$ ( 28 out of 83 patients), and the nonresponse rate (stable disease + progression disease) was $66 \%$ ( 55 of 83 patients).

To investigate whether BC FOXA1 expression was correlated with response to chemotherapy, the patients were divided into 2 groups (FOXA1 high and FOXA1 low) based on the higher or lower FOXA1 expression level in their BC tissue. The main clinical manifestations demonstrated that there were no differences in age and sex in the 2 groups of patients. A comparison of the 2 groups demonstrated a statistically significant difference in the distribution of tumor-infiltrating levels ( $\mathrm{T}$ stage). 
Fig. 1. Comparison of infiltration of CTL, M2 TAM, and DC between FOXA1-low and FOXA1-high groups in bladder cancer. a Patients were divided into 2 groups by the FOXA1 expression level. High expression: intense staining of tumor cells (left panel) and low expression = no or faint staining of tumor cells (right). b FOXA1low groups (right panel) showed significantly higher infiltration of CD8+ CTL, lower infiltration of CD163+ M2 TAM compared with the FOXA1-high groups (left panel). Scale bar, $50 \mu \mathrm{m}$. CTL, cytotoxic T lymphocyte; TAM, tumor-associated macrophage; DC, dendritic cell. ${ }^{* *} p<$ 0.01 .

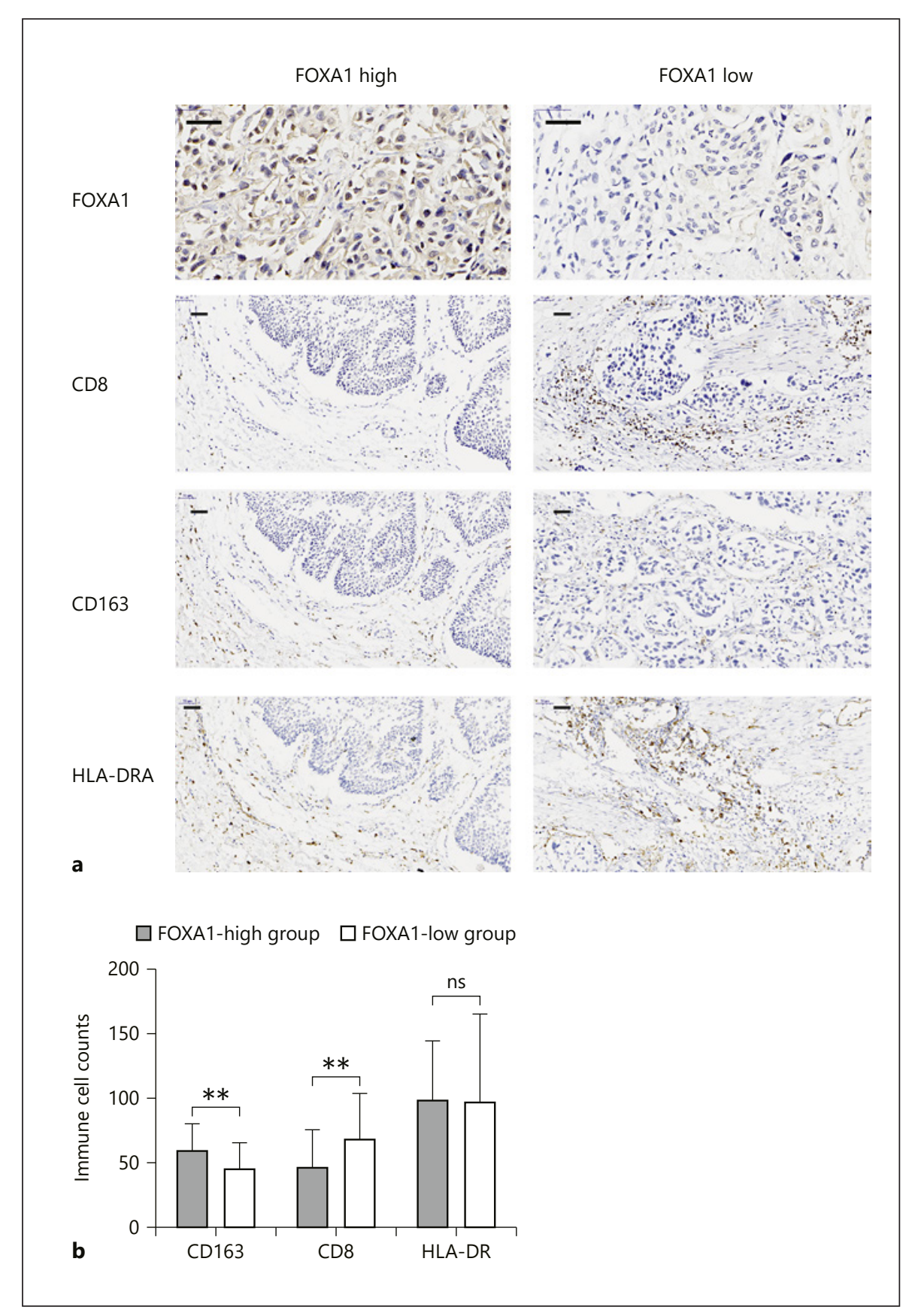

Fifty-six percent of patients had T4 tumors in the FOXA1low group, whereas $30 \%$ of patients had T4 tumors in the FOXA1-high group. The patients who had positive lymph node or lymphadenopathy on radiographic imaging were $70 \%$ and $53 \%$ in the FOXA1-low group and the FOXA1high group, respectively (Table 2 ). These results show that lower level of tumor FOXA1 expression was associated with higher grade BC. The frequency of the clinical responses to chemotherapy demonstrated a significant difference in the 2 groups of patients. In the FOXA1-low group, the frequency of objective response (complete + partial regression) constituted 58\%. In the FOXA1-high group, the objective response rate was $12 \%$, and $88 \%$ of patients were resistant to chemotherapy (Table 2).

Immunohistochemical (IHC) staining of CD8 was used as a marker for CTL, CD163 for M2 TAM, and HLADR for DC. In tumors with higher infiltration of the M2 TAM group (proportion $>3 \%$ ), the probability of respond- 
Table 1. Clinical parameters of $B C$ patients who received chemotherapy

\begin{tabular}{ll}
\hline Clinical parameters & $N(\%)$ \\
\hline Sex & \\
Male & $68(82)$ \\
Female & $15(18)$ \\
Age & \\
$\quad<69$ years & $27(33)$ \\
$\geq 69$ years & $56(67)$ \\
T stage & $16(19)$ \\
T2 & $32(39)$ \\
T3 & $35(42)$ \\
T4 & \\
N stage & $32(39)$ \\
N0 & $51(61)$ \\
N+ & \\
Treatment & $24(29)$ \\
NAC & $59(71)$ \\
Chemotherapy & \\
Clinical response & $28(34)$ \\
CR + PR & $55(66)$ \\
SD + PD &
\end{tabular}

BC, bladder cancer; NAC, neoadjuvant chemotherapy; CR, complete response; $\mathrm{PR}$, partial response; $\mathrm{SD}$, stable disease; $\mathrm{PD}$, progression disease.

Table 3. The correlation between immune cell infiltration and clinical response to chemotherapy

\begin{tabular}{|c|c|c|c|}
\hline \multirow[t]{2}{*}{ Immune cell } & \multicolumn{2}{|c|}{ Clinical response } & \multirow[t]{2}{*}{$p$ level } \\
\hline & $\mathrm{CR}+\mathrm{PR}, n(\%)$ & $\mathrm{SD}+\mathrm{PD}, n(\%)$ & \\
\hline \multicolumn{4}{|c|}{ M2 TAM proportion } \\
\hline$<3 \%$ & $19(46)$ & $22(54)$ & \multirow{2}{*}{0.02} \\
\hline$>3 \%$ & $9(21)$ & $33(79)$ & \\
\hline \multicolumn{4}{|c|}{ CD8+ CTL proportion } \\
\hline$<5 \%$ & $7(17)$ & $34(83)$ & \multirow{3}{*}{0.002} \\
\hline$>5 \%$ & $21(50)$ & $21(50)$ & \\
\hline \multicolumn{3}{|l|}{$\begin{array}{l}\text { HLA-DR+ DC } \\
\text { proportion }\end{array}$} & \\
\hline$>8 \%$ & $13(45)$ & $29(55)$ & \multirow{2}{*}{0.65} \\
\hline$<8 \%$ & $15(37)$ & $26(63)$ & \\
\hline
\end{tabular}

$p$ level $-x^{2}$ test. $C R$, complete response; $P R$, partial response; $S D$, stable disease; $P D$, progression disease; TAM tumor-associated macrophage; CTL, cytotoxic T lymphocyte; DC, dendritic cell.

ers (complete response + partial response) was significantly lower compared with lower infiltration of the M2 TAM group (proportion $<3 \%)(21 \%$ vs. $46 \%, p=0.02)$. For CTL, the higher infiltration group had a higher probabil-

FOXA1 and Tumor-Infiltrating Immune

Cells and Effect of Chemotherapy
Table 2. Clinical parameters of patients of FXOA1-low and FOXA1high groups

\begin{tabular}{|c|c|c|c|}
\hline Clinical parameters & $\begin{array}{l}\text { FOXA1-low } \\
\text { group, } n(\%)\end{array}$ & $\begin{array}{l}\text { FOXA1-high } \\
\text { group, } n(\%)\end{array}$ & $p$ level \\
\hline \multicolumn{4}{|l|}{ Sex } \\
\hline Male & $32(80)$ & $36(84)$ & \multirow{2}{*}{0.66} \\
\hline Female & $8(20)$ & $7(16)$ & \\
\hline \multicolumn{4}{|l|}{ Age } \\
\hline$<69$ years & $14(35)$ & $13(30)$ & \multirow{2}{*}{0.64} \\
\hline$\geq 69$ years & $26(65)$ & $30(70)$ & \\
\hline \multicolumn{4}{|l|}{ T stage } \\
\hline T2 & $3(8)$ & $13(30)$ & \multirow{3}{*}{0.03} \\
\hline T3 & $15(36)$ & $17(40)$ & \\
\hline T4 & $22(56)$ & $13(30)$ & \\
\hline \multicolumn{4}{|l|}{$\mathrm{N}$ stage } \\
\hline NO & $12(30)$ & $20(47)$ & \multirow[b]{2}{*}{0.12} \\
\hline $\mathrm{N}+$ & $28(70)$ & $23(53)$ & \\
\hline \multicolumn{4}{|l|}{ Clinical response } \\
\hline$C R+P R$ & $23(58)$ & $5(12)$ & \multirow{2}{*}{0.01} \\
\hline$S D+P D$ & $17(42)$ & $38(88)$ & \\
\hline
\end{tabular}

$p$ level $-X^{2}$ test. $C R$, complete response; $P R$, partial response; $S D$, stable disease; PD, progression disease.

Table 4. The correlation between combined FOXA1 expression, M2 infiltration, and CTL infiltration and clinical response to chemotherapy

\begin{tabular}{llll}
\hline $\begin{array}{l}\text { Clinical } \\
\text { response }\end{array}$ & $\begin{array}{l}\text { FOXA1-low, M2 TAM } \\
\text { proportion }<3 \%, C T L \\
\text { proportion }>5 \%, n(\%)\end{array}$ & $\begin{array}{l}\text { The remaining } \\
\text { cases, } n(\%)\end{array}$ & $p$ level \\
\hline CR + PR & $18(82)$ & $10(16)$ & $<0.0001$ \\
SD + PD & $4(18)$ & $51(84)$ & \\
\hline
\end{tabular}

$p$ level $-X^{2}$ test. $C R$, complete response; $P R$, partial response; $S D$, stable disease; PD, progression disease; TAM tumor-associated macrophage; CTL, cytotoxic T lymphocyte.

ity of responders (proportion $>5 \%$ group: $50 \%$; proportion $<5 \%: 17 \%$; $p=0.002)$. HLA-DR+DC showed no significant differences (Table 3 ). In the FOXA1-low group, 18 out of 42 tumors were found to have M2 TAM proportion $<3 \%$ and CTL proportion $>5 \%$, simultaneously. The objective response rate of this group (FOXA1-low, M2 TAM proportion $<3 \%$, and CTL proportion $>5 \%$ ) was significantly higher compared with the rest of the cases $(82 \%$ vs. $16 \%, p<0.0001$ ) (Table 4). Furthermore, we examined 
the correlation between FOXA1 expression and M2 TAM and CTL infiltration. The results showed that low FOXA1 expression is significantly associated with lower infiltration of M2 TAM and higher infiltration of CTL (Fig. 1b).

\section{Discussion}

In this study, we demonstrated the indicative value of FOXA1 expression level, tumor-infiltrated CTL, and M2 TAM for the efficiency of cisplatin-based chemotherapy in BC. The correlation of FOXA1 expression level and immune cell infiltration was also investigated. The forkhead box (FOX) superfamily of transcription factors plays an essential role in human bladder malignant progression $[14,15]$. FOXA1, a member of the FOX superfamily, was found to be differentially expressed in various $\mathrm{BC}$ molecular subtypes and was involved in urothelial cell differentiation [16]. The TCGA database identified 5 subtypes of MIBC based on specific gene mRNA expression profiles. The subtypes of MIBC were luminal, luminal-infiltrated, basal-squamous, neuronal, and luminal-papillary. Luminal and basal were confirmed to be the 2 major subtypes among these 5 subtypes. Elevated FOXA1 expression was associated with the luminal molecular subtype, whereas decreased FOXA1 expression was associated with the basal-squamous subtype which is more sensitive to chemotherapy compared to the luminal MIBC. In our results, the FOXA1-low group was associated with a higher tumor stage and a higher frequency of positive lymph node. The clinical response to chemotherapy in 2 groups had also a significant difference: in the FOXA1-low group, the response was $58 \%$, while in the FOXA1-high group only $12 \%$. These results indicated that the FOXA1 expression level has a potential role in assessing tumor prognosis and chemotherapy efficiency in BC.

$\mathrm{BC}$ has long been strongly related to immune cell infiltration [17]. Notably, tumor-infiltrated immune cells are associated with significantly worse OS $[18,19]$. TAM and tumor-infiltrated CTL are key cells of immunity that control intratumoral immune status, tumor growth, and metastasis and can also contribute to the response to chemotherapy and immunotherapy [20-22]. M2 macrophage polarization often occurs in tumors after chemotherapy and facilitates resistance [23]. M2 TAM-induced resistance to chemotherapy can be explained by activation of the IL-10/ STAT3/BCL-2 signal transduction pathway. IL-10, secreted by M2 TAM, increases the expression of the anti-apoptotic gene BCL-2, leading to the development of resistance in tumor cells $[24,25]$. We found that tumors with lower levels of infiltrated M2 TAM (proportion $<3 \%$ ) responded to chemotherapy with $46 \%$ probability, while in tumors with higher levels of infiltrated M2 TAM (proportion $>3 \%$ ), the probability of response to chemotherapy was significantly lower (21\%). For the CTL, the probability of response to chemotherapy in tumors with a higher level of infiltrated CTL (proportion $>5 \%$ ) was $50 \%$ and in tumors with a lower level of infiltrated CTL (proportion $<5 \%$ ) was $17 \%$. For DCs, there was no significant difference between tumors with a higher or lower level of infiltrated DCs. These data indicated that M2 TAM and CTL are potential independent predictors for chemotherapy in $\mathrm{BC}$.

Basal-subtype MIBC shows a characteristic "hot" immunophenotype (an abundance of CTLs) and lower FOXA1 expression, as compared with luminal-subtype MIBC. To investigate the correlation between FOXA1 expression and immune-infiltrating cells in MIBC, IHC was conducted and showed that FOXA1 expression correlates with the infiltration of M2 TAM and CTL. The mechanism of how FOXA1 regulates the immune microenvironment needs to be further investigated. Also, we found that tumors in the FOXA1-low group with a lower level of M2 TAM (proportion $<3 \%$ ) and a higher level of CTL (proportion $>5 \%$ ) responded to chemotherapy with $82 \%$.

Thus, we have shown that lower expression of FOXA1 correlates with a better response rate to chemotherapy, lower infiltration of M2 TAM, and higher infiltration of CTL. Detecting FOXA1 expression combined with infiltrating M2 TAM and CTL by IHC is a promising strategy to predict the efficiency of chemotherapy in BC.

\section{Statement of Ethics}

This study was approved by the Ethics Committee of Zhongda Hospital, Southeast University (Approval No. 2018ZDKYSB102). All subjects provided written informed consent following the Declaration of Helsinki.

\section{Conflict of Interest Statement}

The authors report no conflicts of interest.

\section{Funding Sources}

This work was supported by the National Natural Science Foundation of China (Nos. 81872089, 81370849, 81672551, 81300472, 81070592, 81202268, and 81202034), Natural Science Foundation of Jiangsu Province (BK20161434), and Nanjing Science Development Project (YKK17289). 


\section{Author Contributions}

Yiduo Wang and Ming Chen designed the study; Yiduo Wang and Huan Qu wrote the manuscript; Ming Chen, Yiduo Wang, Bin $\mathrm{Xu}$, Jianping $\mathrm{Wu}$, and Shuqiu Chen performed the surgeries; Yiduo Wang, Kai Lu, and Chunhui Liu analyzed the data; Yiduo Wang and Huan Qu conducted the IHC.

\section{Data Availability Statement}

All data generated or analyzed during this study are included in this article. Further inquiries can be directed to the corresponding author.

\section{References}

1 Ferlay J, Colombet M, Soerjomataram I, Dyba T, Randi G, Bettio M, et al. Cancer incidence and mortality patterns in Europe: estimates for 40 countries and 25 major cancers in 2018. Eur J Cancer. 2018;103:356-87.

2 Burger M, Catto JW, Dalbagni G, Grossman HB, Herr H, Karakiewicz P, et al. Epidemiology and risk factors of urothelial bladder cancer. Eur Urol. 2013;63(2):234-41.

3 Abdollah F, Gandaglia G, Thuret R, Schmitges J, Tian Z, Jeldres C, et al. Incidence, survival and mortality rates of stage-specific bladder cancer in United States: a trend analysis. Cancer Epidemiol. 2013;37(3):219-25.

4 Dall'Era MA, Cheng L, Pan CX. Contemporary management of muscle-invasive bladder cancer. Expert Rev Anticancer Ther. 2012; 12(7):941-50.

5 Sternberg CN, de Mulder P, Schornagel JH, Theodore C, Fossa SD, van Oosterom AT, et al. Seven year update of an EORTC phase III trial of high-dose intensity M-VAC chemotherapy and G-CSF versus classic M-VAC in advanced urothelial tract tumours. Eur J Cancer. 2006;42(1):50-4.

6 Sternberg CN, Donat SM, Bellmunt J, Millikan RE, Stadler W, De Mulder P, et al. Chemotherapy for bladder cancer: treatment guidelines for neoadjuvant chemotherapy, bladder preservation, adjuvant chemotherapy, and metastatic cancer. Urology. 2007; 69(Suppl 1):62-79.

7 Mooney D, Paluri R, Mehta A, Goyal J, Sonpavde G. Update in systemic therapy of urologic malignancies. Postgrad Med. 2014; 126(1):44-54.

8 Bajorin DF, Dodd PM, Mazumdar M, Fazzari M, McCaffrey JA, Scher HI, et al. Long-term survival in metastatic transitional-cell carcinoma and prognostic factors predicting outcome of therapy. J Clin Oncol. 1999;17(10): 3173-81.
9 Yafi FA, North S, Kassouf W. First- and second-line therapy for metastatic urothelial carcinoma of the bladder. Curr Oncol. 2011; 18(1):e25-34.

10 Galsky MD, Hahn NM, Powles T, Hellerstedt BA, Lerner SP, Gardner TA, et al. Gemcitabine, Cisplatin, and sunitinib for metastatic urothelial carcinoma and as preoperative therapy for muscle-invasive bladder cancer. Clin Genitourin Cancer. 2013;11(2): 175-81.

11 Robertson AG, Kim J, Al-Ahmadie H, Bellmunt J, Guo G, Cherniack AD, et al. Comprehensive molecular characterization of muscle-invasive bladder cancer. Cell. 2018;174(4): 1033.

12 Fridman WH, Pagès F, Sautès-Fridman C, Galon J. The immune contexture in human tumours: impact on clinical outcome. Nat Rev Cancer. 2012;12(4):298-306.

13 Sharma P, Shen Y, Wen S, Yamada S, Jungbluth AA, Gnjatic S, et al. CD8 tumor-infiltrating lymphocytes are predictive of survival in muscle-invasive urothelial carcinoma. Proc Natl Acad Sci USA. 2007;104(10):396772.

14 Jackson BC, Carpenter C, Nebert DW, Vasiliou V. Update of human and mouse forkhead box (FOX) gene families. Hum Genomics. 2010;4(5):345-52.

15 Hannenhalli S, Kaestner KH. The evolution of Fox genes and their role in development and disease. Nat Rev Genet. 2009;10(4):233-40.

16 DeGraff DJ, Cates JM, Mauney JR, Clark PE, Matusik RJ, Adam RM. When urothelial differentiation pathways go wrong: implications for bladder cancer development and progression. Urol Oncol. 2013;31(6):802-11.
17 Masson-Lecomte A, Rava M, Real FX, Hartmann A, Allory Y, Malats N. Inflammatory biomarkers and bladder cancer prognosis: a systematic review. Eur Urol. 2014;66(6): 1078-91.

18 Aljabery F, Olsson H, Gimm O, Jahnson S, Shabo I. M2-macrophage infiltration and macrophage traits of tumor cells in urinary bladder cancer. Urol Oncol. 2018;36(4):159e26.

19 Hodgson A, Xu B, Satkunasivam R, Downes MR. Tumour front inflammation and necrosis are independent prognostic predictors in high-grade urothelial carcinoma of the bladder. J Clin Pathol. 2018;71(2):154-60.

20 Stakheyeva M, Riabov V, Mitrofanova I, Litviakov N, Choynzonov E, Cherdyntseva N, et al. Role of the immune component of tumor microenvironment in the efficiency of cancer treatment: perspectives for the personalized therapy. Curr Pharm Des. 2017;23(32):480726.

21 Riabov V, Gudima A, Wang N, Mickley A, Orekhov A, Kzhyshkowska J. Role of tumor associated macrophages in tumor angiogenesis and lymphangiogenesis. Front Physiol. 2014;5:75.

22 Noy R, Pollard JW. Tumor-associated macrophages: from mechanisms to therapy. Immunity. 2014;41(1):49-61

23 Zitvogel L, Galluzzi L, Smyth MJ, Kroemer G. Mechanism of action of conventional and targeted anticancer therapies: reinstating immunosurveillance. Immunity. 2013;39(1):74-88.

24 De Palma M, Lewis CE. Cancer: macrophages limit chemotherapy. Nature. 2011;472(7343): 303-4.

25 Hughes R, Qian BZ, Rowan C, Muthana M, Keklikoglou I, Olson OC, et al. Perivascular M2 macrophages stimulate tumor relapse after chemotherapy. Cancer Res. 2015;75(17): 3479-91. 